\title{
PET-CT evaluation of the curative effect of
}

\section{crizotinib on malignant myofibroblastoma with rare mutation of ALK R40I: a case report and literature review}

OncoTargets and Therapy

\section{Li Yangl,* \\ Yufeng $\mathrm{Wu}^{1, *}$ \\ Hong Tang' \\ Jiuzhou Zhao ${ }^{2}$ \\ Dongdong Zhao' \\ Sen Yang' \\ Qiming Wang'}

'Department of Internal Medicine,

Affiliated Cancer Hospital of

Zhengzhou University, Henan

Cancer Hospital, Zhengzhou, China;

${ }^{2}$ Department of Molecular Pathology,

Affiliated Cancer Hospital of

Zhengzhou University, Henan Cancer

Hospital, Zhengzhou, China

*These authors contributed equally to this work
Correspondence: Qiming Wang Department of Internal Medicine, Affiliated Cancer Hospital of Zhengzhou University, Henan Cancer Hospital, No 127 Dongming Road, Zhengzhou 450008, China

Fax +86 37I 65588134

Email qimingwang1006@gmail.com

\begin{abstract}
Objective: The purpose of this article is to explore the targeted treatment of malignant myofibroblastoma and evaluate the role of neoplasm metabolite markers in the evaluation of efficacy after targeted therapy.

Method: This report described a case of myofibroblastic sarcoma with rare mutation of $A L K$ $R 401$ in a 58-year-old man prescribed with crizotinib, to evaluate its curative effect by positron emission tomography coupled with computed tomography (PET-CT). After the progressive disease in the brain, bevacizumab combined with crizotinib was administered. The Response Evaluation Criteria in Solid Tumors (RECIST), metabolic tumor volume (MTV), and total lesion glycolysis (TLG) were used to assess the efficacy. The efficacy was assessed by comparing changes in MTV and TLG.
\end{abstract}

Result: After the treatment of crizotinib, the tumor volume was decreased. However, bevacizumab combined with crizotinib had not improved the prognosis. The change of MTV and TLG was consistent with the efficacy. The increase of MTV and TLG is an early indicator of the poor prognosis of patients.

Conclusion: The treatment of the crizotinib patient with the mutation of ALK R 401 was effective. The values of MTV and TLG reflected the prognosis earlier than RECIST.

Keywords: malignant myofibroblastoma, PET-CT, ALK R401, volume-dependent parameter

\section{Introduction}

Malignant myofibroblastoma is a rare tumor in adults and pediatric patients, ${ }^{1-4}$ the classification of which belongs to myofibroblastic tumors. The classification of the myofibroblastic tumors is myfibroblastic sarcoma (MS), inflammatory myofibroblastic, myofibromatosis, myofibroblastoma, myopericytoma, and angiomyofibrolastoma. This report is based on low-grade fibrous myofibroblasts, which are infrequently accompanied by metastasis, and surgical treatment is common. It has been known to arise mainly in the head and neck regions and the soft tissues of the extremities, ${ }^{5,6}$ although it could be rarely found at the bone and breast; only a small number of cases have been reported in the literature worldwide. ${ }^{7}$ Inflammatory myofibroblastic tumor (IMT) and MS belong to soft tissue tumors, but the malignant level was different. The expression of anaplastic lymphoma kinase (ALK) protein occurs in $50 \%-70 \%$ of IMTs,${ }^{8,9}$ while the ALK of MS are mostly immunonegative. ${ }^{10,11}$ ALK-R401 is a nonsense mutation, and its function is unknown. ${ }^{12}$ In this case, the immunohistochemistry (IHC) of our patient did not reveal $A L K$ gene mutation, but 
next-generation sequencing (NGS) supported $A L K R 401$ mutation in exon five. Treatment with an ALK inhibitor was found to be effective in reducing tumor size and tumor metabolism.

Tumor-response assessments are still using Response Evaluation Criteria in Solid Tumors (RECIST), which evaluate the effectiveness using the maximum and rear diameters. ${ }^{13-15}$ Although tumor size changes often take time (up to several months), it is necessary in the clinical treatment process to be able to predict early clinical response and to adjust treatment plans accordingly. Furthermore, metabolic changes in the tumor may reflect changes in the condition earlier. Positron emission tomography coupled with computed tomography (PET-CT) has long been a reference imaging tool for the diagnosis and staging of tumors, ${ }^{16}$ particularly in cases where the metastatic tumors of the primary lesion were unknown. ${ }^{17}$ There are some studies confirming that metabolic tumor volume (MTV) and total lesion glycolysis (TLG) as a tumor metabolic index can be used to assess the prognosis of patients. ${ }^{18}$ Herein, we reviewed the use of PET-CT to assess treatment response and found that the MTV can reflect the metabolic and volume changes, which are more conducive to predict the prognosis of patients.

\section{Case report}

A 58-year-old male presented to the hospital with paroxysmal pain in the chest and discomfort in the head on August 10, 2015. The Karnofsky performance status (KPS) score was 60. PET-CT revealed a right upper chest mass was $51 \times 41 \mathrm{~mm}$, with a maximum standardized uptake value (SUV) of 5.6, accompanied with brain, bone, liver, and right subscapularis muscle metastases with high SUV. We recorded the tumor size of the primary tumor and metastases. The specimens showed malignant spindle cells, which displayed immunohistochemical positive staining for CK, SMA, CD34, CD99, and Bcl-2, and negative for Des, S-100, and B-Catenin. ALK was not supported (Ventana IHC) (Figure 1). The patient was then diagnosed with malignant myofibroblastoma with brain, bone, and liver metastases.

Considering the patient's poor KPS score (60 at diagnosis) and metastases all over the body, chemotherapy, radiochemotherapy, or surgery was excluded. Methylprednisolone was given at $800 \mathrm{mg} / \mathrm{d}$, day (d)1-5 started from August 20, 2015. The patient then developed an aggravating headache. Fifteen days later, PET-CT imaging showed tumor volume enlargement. The MTV and TLG values are also increased. Subsequently, genetic tests revealed the fifth exon $R 401$ mutation of the $A L K$ gene. Considering the tumor histological and biological features, crizotinib was given at $250 \mathrm{mg}$ daily from September 10, 2015. After 1 month of crizotinib at $250 \mathrm{mg}$ daily, the headache was eased, and PET-CT (October 9, 2015) showed a dramatic reduction in tumor size and metabolism, resulting in stable disease (SD), based on criteria in RECIST 1.1 with KPS score of 70. Conversely, the MTV and TLG values were also significantly reduced.

Two months later, the patient had headaches again, so we reviewed his PET-CT on December 7, 2015. The brain imaging showed that the primary tumor was enlarged with edema, and new metastases could be found in the lung (Figure 2). The tumor size of the right upper chest wall, liver, and right subscapularis muscle metastases were stable, while the MTV and TLG values of them were almost doubled, representing progressive disease. The patient's family rejected the second-generation ALK inhibitors. Thus, bevacizumab (500 mg/d, d1, every 21 days), combined with crizotinib at

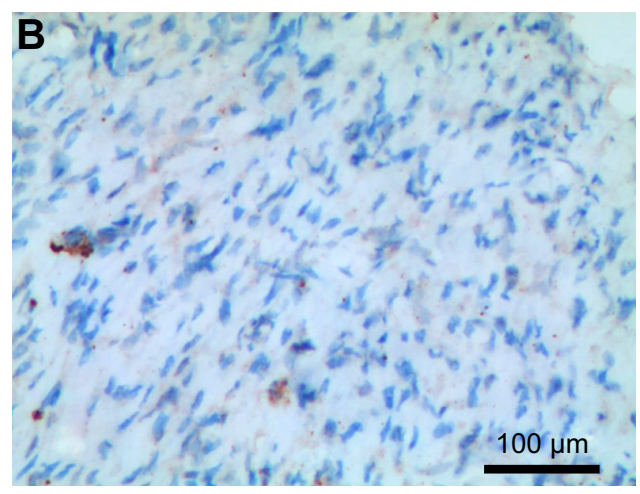

Figure I Histopathological and immunohistological findings of the lymph nodes tissues samples. (A) Hematoxylin and eosin staining showed malignant spindle cells (200x), which displayed immunohistochemical positive staining for CK, SMA, CD34, CD99, and Bcl-2, and negative for Des, S-I00, and B-Catenin. (B) IHC of our patient did not reveal supported ALK gene mutation (200x).

Abbreviations: IHC, immunohistochemistry; ALK, anaplastic lymphoma kinase. 

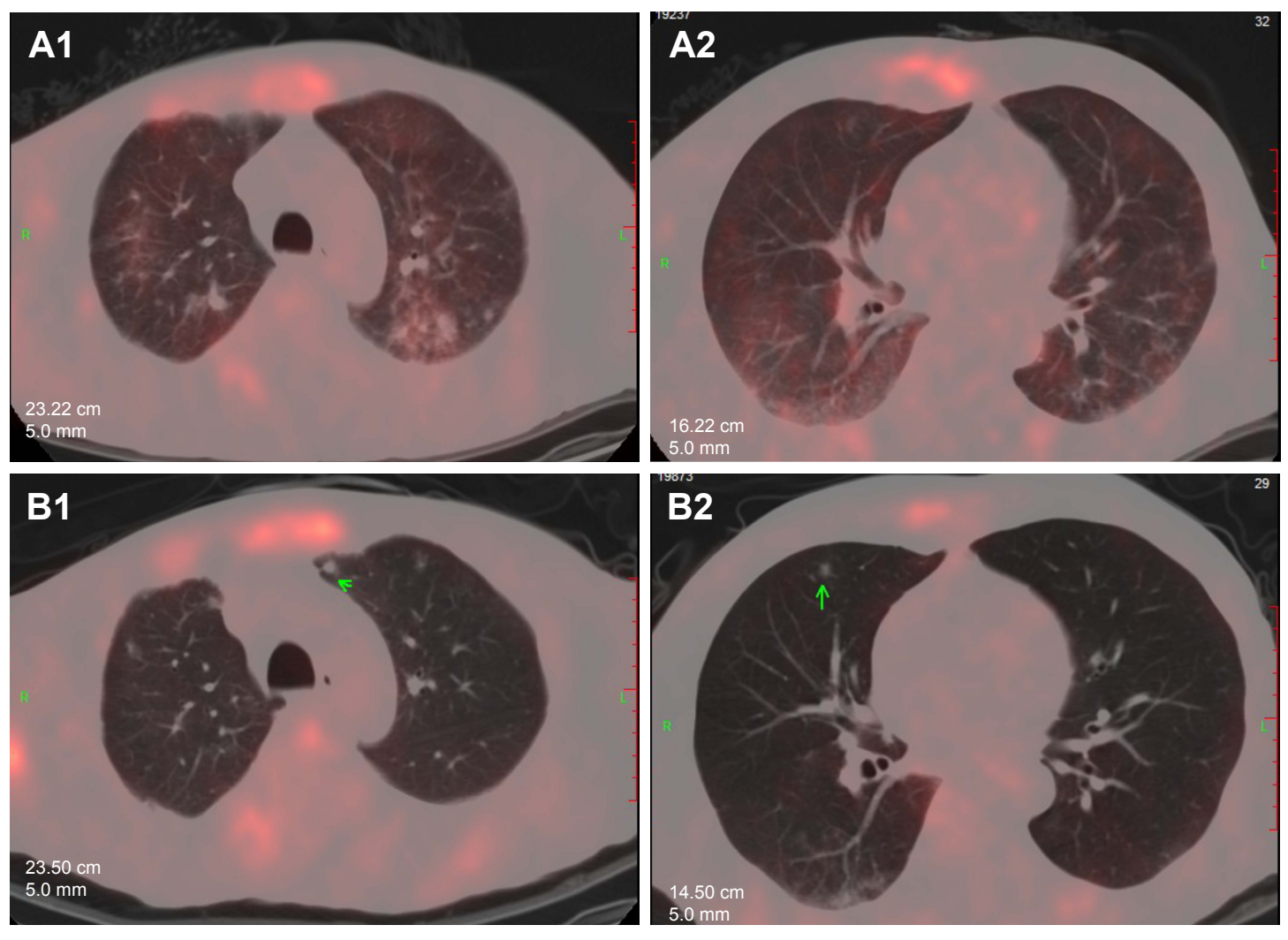

Figure 2 The progression in the lung. (AI and A2) were taken on October 9, 20I5. (BI and B2) were taken on December 10, 20I5, and the green arrows refer to the new lesions.

$250 \mathrm{mg}$ daily, was administered. Twenty days later, the headache was eased, and the KPS score was 80 . Two months after treatment with bevacizumab $(500 \mathrm{mg} / \mathrm{d}, \mathrm{d} 1$, every 21 days $)$ combined with crizotinib $250 \mathrm{mg}$ daily, the headache was aggravated, and the patient developed a poor diet, nausea, and vomiting. The KPS score was 50, PET-CT imaging showed right pleural effusion, all body metastases were enlarged, and the MTV and TLG values were significantly increased, indicating progressive disease (Figure 3). The patient was administered nutritional supportive treatment. Unfortunately, the patient died 1 month later in March 2016 (Figure 4).

Written informed consent was obtained from the patient's wife (the patient's next of kin) for publication of this case report and accompanying images.

\section{Discussion}

Crizotinib is an ATP competitor that inhibits the multitarget protein kinase inhibitor of Met/ALK/ROS. Crizotinib has been used in $A L K$ positive non-small cell lung cancer (NSCLC) to lengthen the period without progression. ALK inhibitors have been reported as effective treatments for $A L K$-rearranged IMT. ${ }^{19,20}$ It was reported that a patient who was diagnosed
$A L K$-1-rearranged IMT with brain metastases was initially treated with crizotinib, and the disease progressed after 3 months. ${ }^{21}$ After that, alectinib, the second-generation ALK inhibitor, was taken as the second-line therapy with 8-month progression-free survival. Our patient had malignant myofibroblastoma with the mutation of $A L K-R 401$. There is no report about the function and treatment effect of the $A L K$ $R 401$, but it was considered implicated in tumorigenesis. In this case, after the treatment of crizotinib, the progression-free survival was 3 months. It has been known that crizotinib has poor central nervous system (CNS) penetration, as evidenced by low concentrations detected in CNS samples during the treatment course, ${ }^{22}$ which was likely the cause of treatment failure in our patient. Also, alectinib, which is a selective ALK inhibitor with high CNS penetration, is active against several secondary mutations that confer acquired resistance to crizotinib. ${ }^{23}$ It may be effective to administer the second generation ALK inhibitor after drug resistance.

Bevacizumab, a recombinant, humanized monoclonal antibody against vascular endothelial growth factor (VEGF), is a vascular-targeted therapy that may inhibit neovascularization, ${ }^{24}$ such as slower tumor development, reduced metastasis development, and improved drug 


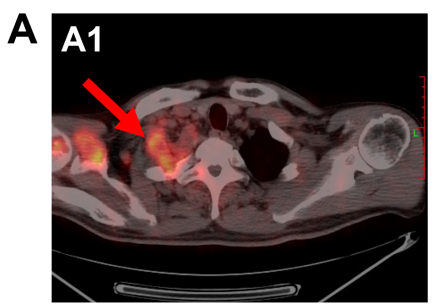

B B1

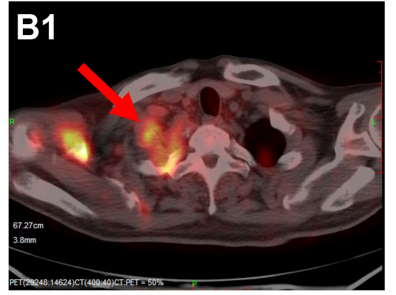

C $\mathbf{c 1}$

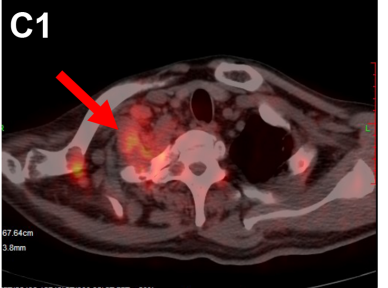

D D1

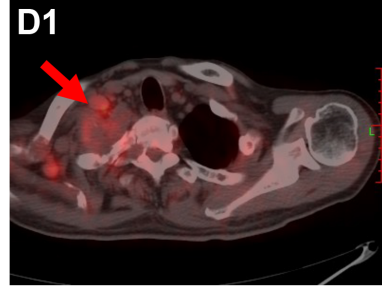

E E1

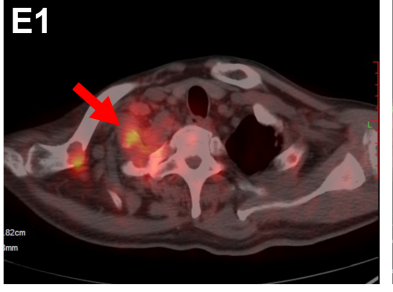

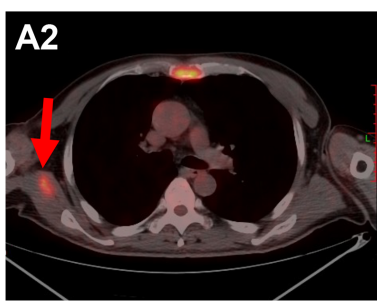
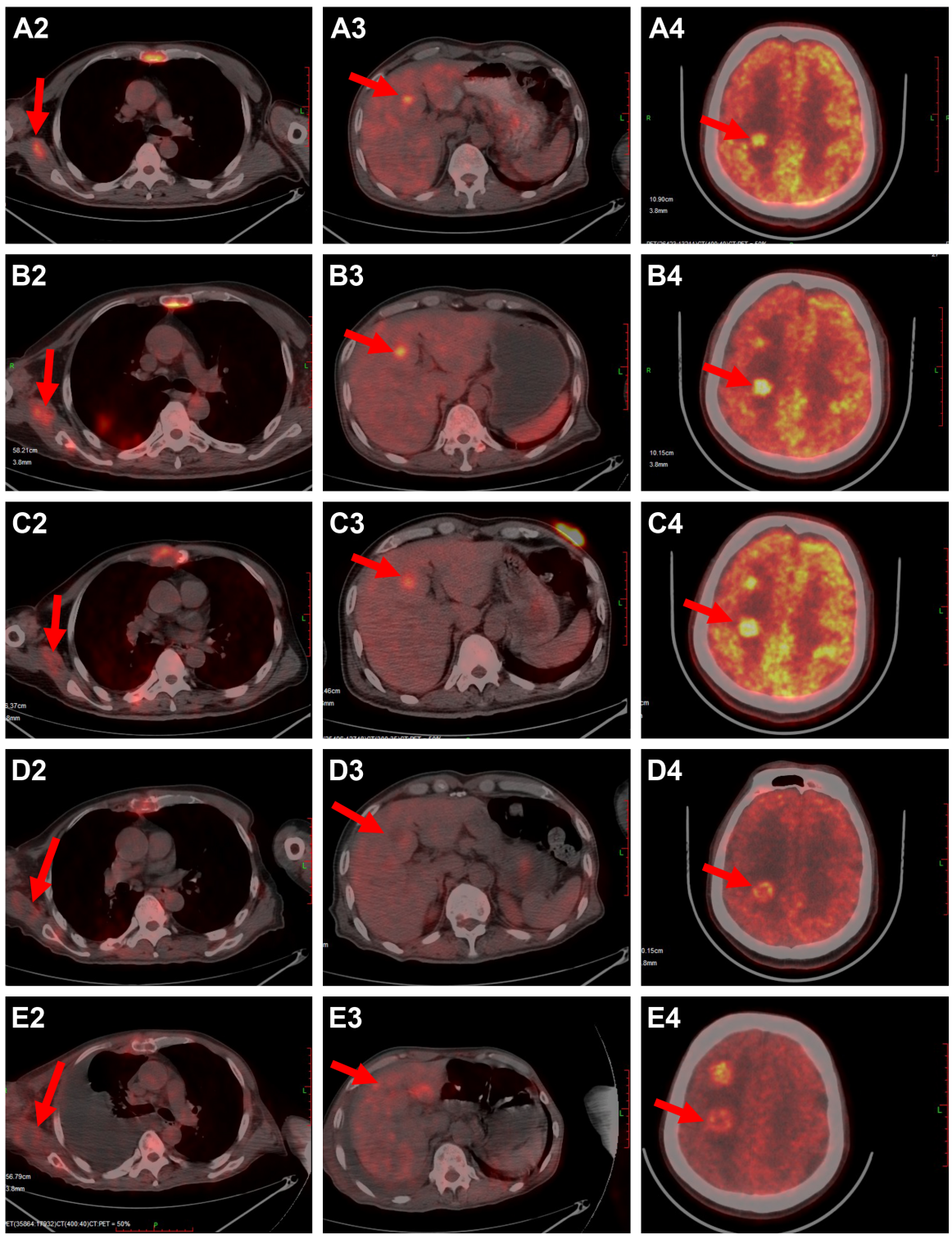

Figure 3 The dynamic evolution of the patient's primary and metastases tumors during the treatment in the PET-CT images. (A) Images AI-A4 were taken at the first clinic visit on August 10,2015. The right upper chest mass was $5 \mathrm{I} \times 4 \mathrm{I} \mathrm{mm}$, with a the maximum of standardized uptake value (SUV ${ }_{\max }$ ) of 5.6, MTV value of 28.8, and TLG value of

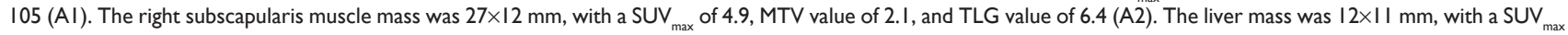
of 6.2, MTV value of 2, and TLG value of 8.2 (A3). The larger mass in the occipital lobe of the brain mass was $26 \times 17 \mathrm{~mm}$ (A4). (B) Images BI-B4 as baseline were taken after the methylprednisolone on September 10, 20I5. The right upper chest mass was $53 \times 52 \mathrm{~mm}$, with a maximum SUV value of 7.2 , MTV value of 39.9 , and TLG value of I74 (BI). The right subscapularis muscle mass was $27 \times 17 \mathrm{~mm}$, with a SUV ${ }_{\max }$ value of 4.8 , MTV value of 5.2 , and TLG value of I5.5 (B2). The liver mass was I2×II mm, with a SUV $_{\max }$ value of 6.7, MTV value of 4, and TLG value of I5.4 (B3). The brain mass was $27 \times$ I8 (B4). (C) Images CI-C4 were taken after I month treatment of oral crizotinib on October 9, 2015. The size of the tumor seemed to be stable. The right upper chest mass was $44 \times 38 \mathrm{~mm}$, with a maximum SUV value of 8 , MTV value of I4.2, and TLG value

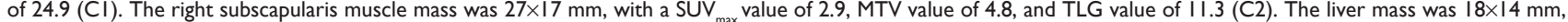
with a SUV ${ }_{\max }$ value of 4, MTV value of 2.2, and TLG value of 6.8 (C3). The brain mass was $27 \times 18 \mathrm{~mm}$ (C4). (D) Images DI-D4 were taken on December I0, 20I5, after 3 months of oral crizotinib, and there was progressive disease in the brain. The right upper chest mass was $46 \times 43$ mm, with a maximum SUV value of 3.6, MTV value of 28.5 , and TLG value of 72.5 (DI). The right subscapularis muscle mass was $28 \times 16 \mathrm{~mm}$, with a SUV ${ }_{\max }$ value of 2.9 , MTV value of 9 , and TLG value of I5.5 (D2). The liver mass was $13 \times 8 \mathrm{~mm}$, with a SUV value of I.7, MTV value of II, and TLG value of I4.I (D3). The brain mass was $28 \times 18 \mathrm{~mm}$ (D4). (E) Images EI-E4 were taken on February 2, 20I6, after two cycles of bevacizumab with oral crizotinib, and all tumors were increased in bulk. The right upper chest mass was $60 \times 56$ mm, with a maximum SUV value of 3 , MTV value of 88.4, and TLG value of 168.6 (EI). The right subscapularis muscle mass was $42 \times 34 \mathrm{~mm}$, with a SUV $\max$ value of 2.4 , MTV value of 49.8 , and TLG value of 73.4 (E2). The liver mass was $17 \times 13 \mathrm{~mm}$, with a SUV max value of 3.5, MTV value of I3.I, and TLG value of 25.2 (E3). The brain mass was $30 \times 19$ mm (E4). The change of the tumor of the right chest wall (AI-EI). The change of the right upper arm muscle space (A2-E2). The change of the liver (A3-E3). The change of the brain (A4-E4).

Abbreviations: PET-CT, positron emission tomography coupled with computed tomography; SUV ${ }_{\text {max }}$, the maximum of standardized uptake value; MTV, metabolic tumor volume; TLG, total lesion glycolysis.

delivery through vascular normalization. ${ }^{25}$ Bevacizumab is more effective when combined with other conventional therapies, such as chemotherapy or radiotherapy, and may be added to treatment regimens to increase their efficacy or to reduce developing therapeutic resistance. ${ }^{26,27}$ Several studies have demonstrated that bevacizumab could reduce brain edema and intracranial pressure, reducing the symptoms of headache or dizziness. Currently, the clinical trials 


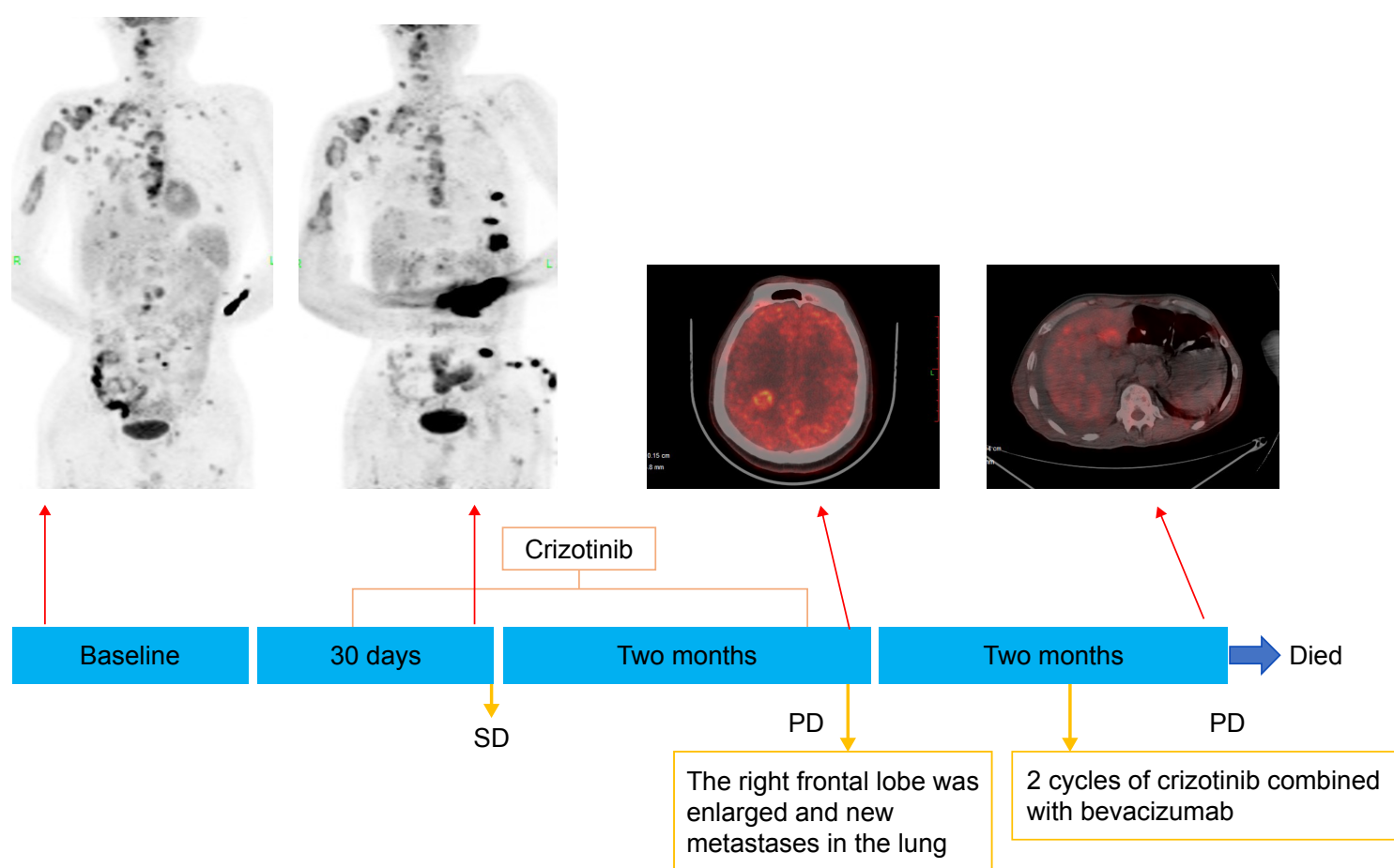

Figure 4 The clinical course of the diagnosis and treatment of the patient. On August II, 20I5, the PET-CT scan of the patient suggested right upper chest, brain, bone, liver, and right subscapularis muscle metastases. Methylprednisolone was given at $800 \mathrm{mg} / \mathrm{d}$, dl-5, started on August 20, 20I5. On September I0, 20I5, PET-CT imaging showed tumor volume enlargement, and crizotinib was given at $250 \mathrm{mg}$ daily. On October 9, 2015 (after one month of crizotinib treatment), PET-CT imaging showed a dramatic reduction in tumor size and metabolism, resulting in SD. (The concentrations in the left elbow and chest wall were radiocontamination.) On December 7 , 20I5, the brain imaging PET-CT showed that the primary tumor was enlarged, representing progressive disease in the brain, and bevacizumab (500 mg/d, $\mathrm{dl}$, every $2 \mathrm{I}$ days), combined with crizotinib at $250 \mathrm{mg}$ daily, was administered. On February 2, 2016 (after two cycles of bevacizumab with crizotinib), PET-CT imaging showed right pleural effusion, all body metastases were enlarged, and pleural cavity and peritoneal effusions could be found. Then, nutritional supportive treatment was given. The patient died in March 2016.

Abbreviations: PET-CT, positron emission tomography coupled with computed tomography; PD, progressive disease; SD, stable disease.

of the EGFR-TKI (Epidermal Growth Factor ReceptorTyrosine Kinase Inhibitor), combined with bevacizumab, have shown good curative effects in the treatment of lung adenocarcinoma. ${ }^{28,29}$ So, in our treatment, when the disease progressed after oral crizotinib, bevacizumab combined with crizotinib was given. Then the headache was eased transient, but the treatment effect was unsatisfactory. Whether bevacizumab combined with an ALK inhibitor is efficacious is worth exploring.

PET-CT is not only a reference imaging tool for the diagnosis and staging of tumors, but can also reflect the metabolic status of the tumor, to be used for evaluation of efficacy and prognosis. ${ }^{30}$ Currently, the prognostic information was deduced from the SUV, MTV, and TLG, which were significantly associated with an early metabolic response. ${ }^{31}$ Additionally, once PET-CT was used to evaluate the effects of a patient with multiple metastases, clinicians can comprehensively assess patient's condition changes. In targeted therapy, PET-CT can reflect the metabolic abilities of the cells to predict the curative effect early and adjust the treatment plan in time. At present, MTV is considered a prognostic indicator of tumor survival, being a volumetric and metabolic biomarker of the tumor. ${ }^{32}$ Thus, unlike $\mathrm{SUV}_{\max }$, MTV can quantify the overall tumor burden. In some studies, higher MTV and TLG were significantly associated with shorter progression-free survival (PFS). In this case, the multiple growth of MTV may be related to the poor prognosis of the tumor.

\section{Conclusion}

Targeted therapy can be considered in the treatment of the poor KPS scores patient of advanced tumor with multiple metastases. Patients with mutated ALK-R401 are effective in the treatment of crizotinib. However, crizotinib has low penetration into the central nervous system, patient with head metastases is responded poorly to it. Bevacizumab, combined with crizotinib, had not improved the prognosis. Bevacizumab combined with crizotinib had not achieved excellent outcome in this advanced tumor patient with head metastases. PET-CT can be used to comprehensively assess the condition of patients with advanced tumor. MTV and TLG can reflect the metabolic changes of the tumor, and the sudden increase in its value may be related to the poor prognosis of the patient. 


\section{Acknowledgments}

This study was supported, in part, by the National Natural Science Foundation of China (No 81272600), a project co-sponsored by the Henan Province and Ministry of Health of Medical Science and Technology Program (No 201601026), "51282 project", leading talent of Henan Provincial Health Science and Technology Innovation Talents (No [2016]32), Natural Science Foundation of Henan Province (No 162300410300), Wu Jieping Medical Foundation for Clinical Research (No 320.6799.15018), Science and Technology Research Program of Henan Province (No 162102310327), Henan Province medical science and technology research project (No 201702249). The funders had no role in the study design, data collection and analysis, decision to publish, or preparation of the manuscript.

\section{Disclosure}

The authors report no conflicts of interest in this work.

\section{References}

1. Hadjigeorgiou GF, Samaras V, Varsos V. Low-grade myofibroblastic sarcoma of the thoracic spine: report of an extreme rare case. $\mathrm{Br} \mathrm{J}$ Neurosurg. 2017;31(6):731-733.

2. Schurch W, Seemayer TA, Gabbiani G. The myofibroblast: a quarter century after its discovery. Am J Surg Pathol. 1998;22(2): 141-147.

3. Jo VY, Fletcher CD. WHO classification of soft tissue tumours: an update based on the 2013 (4th) edition. Pathology. 2014;46(2):95-104.

4. Singh SK, Raheja AA, Jagdevan AK, Singh PK, Purkait S, Suri VA. Malignant temporal muscle myofibroblastoma with an early recurrence in a child. Neurol India. 2017;65(2):408-410.

5. Humphries WE 3rd, Satyan KB, Relyea K, et al. Low-grade myofibroblastic sarcoma of the sacrum. J Neurosurg Pediatr. 2010;6(3): 286-290.

6. Majno G. The story of the myofibroblasts. Am J Surg Pathol. 1979;3(6): $535-542$.

7. Stark M, Hoffmann A, Xiong Z. Mammary myofibrosarcoma: case report and literature review. Breast J. 2011;17(3):300-304.

8. Montgomery EA, Shuster DD, Burkart AL, et al. Inflammatory myofibroblastic tumors of the urinary tract: a clinicopathologic study of 46 cases, including a malignant example inflammatory fibrosarcoma and a subset associated with high-grade urothelial carcinoma. Am J Surg Pathol. 2006;30(12):1502-1512.

9. Sukov WR, Cheville JC, Carlson AW, et al. Utility of ALK-1 protein expression and ALK rearrangements in distinguishing inflammatory myofibroblastic tumor from malignant spindle cell lesions of the urinary bladder. Mod Pathol. 2007;20(5):592-603.

10. Lu ZJ, Zhou SH, Yan SX, Yao HT. Anaplastic lymphoma kinase expression and prognosis in inflammatory myofibroblastic tumours of the maxillary sinus. J Int Med Res. 2009;37(6):2000-2008.

11. Qiu X, Montgomery E, Sun B. Inflammatory myofibroblastic tumor and low-grade myofibroblastic sarcoma: a comparative study of clinicopathologic features and further observations on the immunohistochemical profile of myofibroblasts. Hum Pathol. 2008;39(6):846-856.

12. Yau NK, Fong AY, Leung HF, et al. A pan-cancer review of ALK mutations: implications for carcinogenesis and therapy. Curr Cancer Drug Targets. 2015;15(4):327-336.
13. Choi H. Role of imaging in response assessment and individualised treatment for sarcomas. Clin Oncol (R Coll Radiol). 2017;29(8): 481-488.

14. Riedl CC, Pinker K, Ulaner GA, et al. Comparison of FDG-PET/CT and contrast-enhanced CT for monitoring therapy response in patients with metastatic breast cancer. Eur J Nucl Med Mol Imaging. 2017;44(9): $1428-1437$.

15. Therasse P, Arbuck SG, Eisenhauer EA, et al. New guidelines to evaluate the response to treatment in solid tumors. European Organization for Research and Treatment of Cancer, National Cancer Institute of the United States, National Cancer Institute of Canada. J Natl Cancer Inst. 2000;92(3):205-216.

16. Bang JI, Lim Y, Paeng JC, et al. Comparison of quantitative methods on FDG PET/CT for treatment response evaluation of metastatic colorectal cancer. Nucl Med Mol Imaging. 2017;51(2):147-153.

17. Schafer JF, Gatidis S, Schmidt H, et al. Simultaneous whole-body PET/ MR imaging in comparison to PET/CT in pediatric oncology: initial results. Radiology. 2014;273(1):220-231.

18. Ben Bouallegue F, Tabaa YA, Kafrouni M, Cartron G, Vauchot F, Mariano-Goulart D. Association between textural and morphological tumor indices on baseline PET-CT and early metabolic response on interim PET-CT in bulky malignant lymphomas. Med Phys. 2017;44(9): 4608-4619.

19. Butrynski JE, D'Adamo DR, Hornick JL, et al. Crizotinib in ALKrearranged inflammatory myofibroblastic tumor. N Engl J Med. 2010; 363(18):1727-1733.

20. Liu Q, Kan Y, Zhao Y, He H, Kong L. Epithelioid inflammatory myofibroblastic sarcoma treated with ALK inhibitor: a case report and review of literature. Int J Clin Exp Pathol. 2015;8(11): 15328-15332.

21. Yuan C, Ma MJ, Parker JV, Mekhail TM. Metastatic anaplastic lymphoma kinase-1 (ALK-1)-rearranged inflammatory myofibroblastic sarcoma to the brain with leptomeningeal involvement: favorable response to serial ALK inhibitors: a case report. Am J Case Rep. 2017;18: 799-804.

22. Costa DB, Kobayashi S, Pandya SS, et al. CSF concentration of the anaplastic lymphoma kinase inhibitor crizotinib. J Clin Oncol. 2011; 29(15):e443-e445.

23. Hida T, Nokihara H, Kondo M, et al. Alectinib versus crizotinib in patients with ALK-positive non-small-cell lung cancer (J-ALEX): an open-label, randomised phase 3 trial. Lancet. 2017;390(10089):29-39.

24. Stefanou D, Stamatopoulou S, Sakellaropoulou A, et al. Bevacizumab, pemetrexed and carboplatin in first-line treatment of non-small cell lung cancer patients: focus on patients with brain metastases. Oncol Lett. 2016; 12(6):4635-4642.

25. Aravantinos G, Pectasides D. Bevacizumab in combination with chemotherapy for the treatment of advanced ovarian cancer: a systematic review. J Ovarian Res. 2014;7:57.

26. Horn L, Sandler A. Chemotherapy and antiangiogenic agents in non-small-cell lung cancer. Clin Lung Cancer. 2007;8(Suppl 2): S68-S73.

27. Teng LS, Jin KT, He KF, Wang HH, Cao J, Yu DC. Advances in combination of antiangiogenic agents targeting VEGF-binding and conventional chemotherapy and radiation for cancer treatment. J Chin Med Assoc. 2010;73(6):281-288.

28. Jiang T, Li A, Su C, et al. Addition of bevacizumab for malignant pleural effusion as the manifestation of acquired EGFR-TKI resistance in NSCLC patients. Oncotarget. 2017;8(37):62648-62657.

29. Otsuka K, Hata A, Takeshita J, et al. EGFR-TKI rechallenge with bevacizumab in EGFR-mutant non-small cell lung cancer. Cancer Chemother Pharmacol. 2015;76(4):835-841.

30. Kim KR, Shim HJ, Hwang JE, et al. The role of interim FDG PET-CT after induction chemotherapy as a predictor of concurrent chemoradiotherapy efficacy and prognosis for head and neck cancer. Eur J Nucl Med Mol Imaging. 2018;45(2):170-178. 
31. Matsumoto Y, Baba S, Endo M, et al. Metabolic tumor volume by $18 \mathrm{~F}$ FDG PET/CT can predict the clinical outcome of primary malignant spine/spinal tumors. Biomed Res Int. 2017;2017:8132676.
32. Choi ES, Ha SG, Kim HS, Ha JH, Paeng JC, Han I. Total lesion glycolysis by $18 \mathrm{~F}-\mathrm{FDG} \mathrm{PET} / \mathrm{CT}$ is a reliable predictor of prognosis in soft-tissue sarcoma. Eur J Nucl Med Mol Imaging. 2013;40(12):1836-1842.

\section{Publish your work in this journal}

OncoTargets and Therapy is an international, peer-reviewed, open access journal focusing on the pathological basis of all cancers, potential targets for therapy and treatment protocols employed to improve the management of cancer patients. The journal also focuses on the impact of management programs and new therapeutic agents and protocols on

\section{Dovepress}

patient perspectives such as quality of life, adherence and satisfaction. The manuscript management system is completely online and includes a very quick and fair peer-review system, which is all easy to use. Visit http://www.dovepress.com/testimonials.php to read real quotes from published authors.

Submit your manuscript here: http://www.dovepress.com/oncotargets-and-therapy-journal 